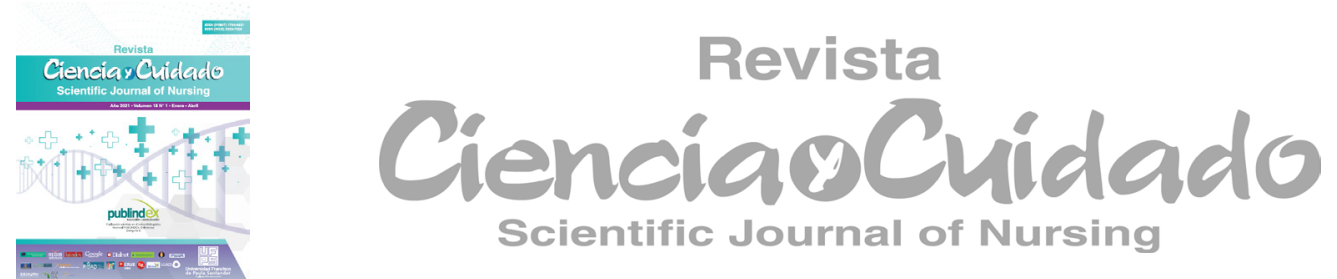

Articulo de Revisión

\title{
Intervenciones educativas para la prevención del pie diabetico
}

\author{
Educational interventions for the prevention of diabetic foot \\ Intervenções educacionais para a prevenção do pé diabético
}

\author{
Mónica Paola Quemba-Mesa ${ }^{1}$ \\ María del Rosario Camargo-Rosas ${ }^{2}$ \\ Nubia Mercedes González-Jiménez ${ }^{3}$
}

\section{Resumen}

Introducción: El pie diabético es una de las complicaciones con mayor impacto en la calidad de vida de las personas con Diabetes Mellitus; en este sentido, las intervenciones educativas son fundamentales en la prevención de esta complicación, por lo cual este artículo tiene el objetivo de caracterizar las intervenciones educativas para el autocuidado y prevención de pie diabético en personas con Diabetes Mellitus. Metodología: Revisión narrativa de la literatura. Se realizó la búsqueda con las siguientes palabras clave: "Diabetes Mellitus and Educative intervention and Diabetic foot and Self-care" en español, inglés y portugués, en las bases de datos Ovid Nursing, LILACS, Google Scholar, Medline y fuentes de literatura gris. Se analizaron 56 artículos que cumplieron los criterios de selección, publicados entre los años 2008 al primer trimestre del 2020. Resultados: Surgieron las siguientes categorías de análisis: 1. Tipos de intervenciones (educación de grupo, educación entre pares, visitas presenciales y telefónicas, redes sociales y comunicación participativa); 2. Ítems a tratar en cada intervención (inspección diaria de los pies, ejercicios y demás cuidados); 3 . Duración de las Intervenciones; y, 4. Impacto de las intervenciones (resultados obtenidos).

Conclusiones: Las diferentes intervenciones educativas al usarse adecuadamente mejoran el nivel de autocuidado de las personas con Diabetes Mellitus y por ende mitigan el desarrollo del pie diabético.

Palabras clave: Diabetes mellitus, intervención educativa, pie diabético, autocuidado, cuidado de enfermería.
Autor de correspondencia*

${ }^{1 *}$ Enfermera, Especialista en Epidemiología Clínica, Magister en Enfermería, Estudiante de Doctorado en Bioética. Docente - Investigadora, Universidad de Boyacá. Tunja Colombia. E-mail: mpquemba@uniboyaca. edu.co. (iD)

${ }^{2}$ Enfermera, Especialista en Gerencia de la Calidad y Auditoria en Servicios de Salud. Docente - Investigadora, Universidad de Boyacá. E-mail: mdcamargo@uniboyaca. edu.co. Tunja - Colombia (iD)

${ }^{3}$ Terapista Respiratoria, Especialista en Gerencia de Instituciones de salud y Magister en Salud Publica. Docente - Investigadora, Universidad de Boyacá. E-mail: nubiagonzalez@uniboyaca.edu.co. Tunja - Colombia.

Recibido: 28 abril 2020

Aprobado: 29 septiembre 2020

Para citar este articulo:

Quemba-Mesa MP, Camargo-Rosas MR, González-Jimenez NM. Creencias frente al embarazo y enfermedadeIntervenciones educativas para la prevención del pie diabético. Rev. cienc. cuidad. 2021;18(1):66-80. https://doi.org/10.22463/17949831.2329

(C) Esta obra está bajo licencia internacional CC-BY-NC-ND (c) $(1) \Theta(\Theta)$ 


\begin{abstract}
Introduction: The diabetic foot is one of the complications with the greatest impact on the quality of life of people with Diabetes Mellitus, being the fundamental educational interventions in the prevention of this complication, for which reason this article aims to characterize the educational interventions for self-care and prevention of diabetic foot in people with Diabetes Mellitus. Methodology: Narrative review of the literature, the search was performed with the keywords "Diabetes Mellitus and Educational intervention and Diabetic foot and Self-care" in Spanish, English and Portuguese, in databases Ovid Nursing, LILACS, Google Scholar, Medline and sources of gray literature, 56 articles that met the selection criteria published between 2008 and the first quarter of 2020 were analyzed. Results: The following categories of analysis emerged: 1 . Types of interventions (group education, peer education, face-to-face and telephone visits, social networks and participatory communication); 2. Items to be treated in each intervention (daily inspection of the feet, exercises and other care); 3. Duration of the Interventions; 4. Impact of the interventions (results obtained). Conclusions: The different educational interventions when used properly improve the level of self-care of people with Diabetes Mellitus and therefore mitigate the development of the diabetic foot.
\end{abstract}

Keywords: Diabetes Mellitus; Educative intervention; Diabetic foot; Self-care; Nursing Care.

\title{
Resumo
}

Introdução: O pé diabético é uma das complicações com maior impacto na qualidade de vida das pessoas com Diabetes Mellitus; neste sentido, as intervenções educativas são fundamentais na prevenção desta complicação. Objetivo: Caracterizar as intervenções educativas para o autocuidado e prevenção do pé diabético em pessoas com Diabetes Mellitus. Materiais e Métodos: Revisão narrativa da literatura. Realizou-se a busca usando as palavras-chave: "Diabetes Mellitus and Educative intervention and Diabetic foot and Self-care" em espanhol, inglês e português, nas bases de dados Ovid Nursing, LILACS, Google Scholar, Medline e fontes de literatura cinzenta. Analisaram-se 56 artigos que seguiram os critérios de seleção, publicados de 2008 ao primeiro trimestre de 2020. Resultados: Surgiram as seguintes categorias de análise: 1. Tipos de intervenções (educação de grupo, educação entre pares, visitas presenciais e telefônicas, redes sociais e comunicação participativa)); 2. Itens a tratar em cada intervenção (inspeção diária dos pés, exercícios e outros cuidados)); 3. Duração das intervenções; e, 4. Impacto das intervenções (resultados obtidos). Conclusões: As diferentes intervenções educativas usando-se adequadamente, melhoram o nível de autocuidado das pessoas com Diabetes Mellitus, portanto reduzem o desenvolvimento do pé diabético.

Palavras-chave: Diabetes Mellitus, intervenção educativa, pé diabético, autocuidado, cuidado de enfermagem.

\section{Introducción}

Ante el aumento de los casos de diabetes mellitus tipo 2 a nivel mundial, se hace necesario buscar medidas que permitan un control glucémico específico y una mejor calidad de vida de las personas, con el fin de reducir la morbilidad y mortalidad a causa de esta patología (12). Teniendo en cuenta que según la OMS cerca de 422 millones de personas en el mundo padecen de Diabetes
Mellitus (DM) (3) y el aumento exponencial de casos nuevos se seguirá observando debido a los hábitos poco saludables que se han adoptado actualmente. Ante esta situación surge la necesidad de educar a los pacientes sobre su enfermedad, para que en conjunto con el personal de salud se evite a toda costa la presencia de complicaciones que disminuyen la calidad de vida de las personas; pero que además acarrean un alto costo a nivel de las Instituciones de salud (4) en los diferentes niveles de atención que se ocupan de aplicar las terapéuticas 
Al respecto, el Pie Diabético (PD) es una de las complicaciones que impacta negativamente tanto a las personas que la padecen como a sus familias, dado que frecuentemente requiere de amputaciones parciales o totales de los miembros inferiores, con la carga psicológica y social que esto conlleva. Carrillo y cols. (5) reportan una prevalencia de PD entre el 8 y el $13 \%$ y se estima que el $25 \%$ de pacientes con DM presentaran una úlcera en el pie durante su vida. A nivel regional, se observa un alto número de amputaciones del pie secundario a la DM, el cual oscila entre el $45 \%$ y el $75 \%$ de todas las causas de amputaciones.

Se debe señalar que las intervenciones educativas juegan un papel muy importante en la prevención del PD, y en general la educación para la salud desempeña una función central entre los componentes de la promoción de la salud. Es por eso que cuando se le enseña al paciente de forma correcta y oportuna la información clave en torno al autocuidado requerido para el manejo de esta patología, ayuda a mejorar su salud y calidad de vida, así como la prevención de las complicaciones crónicas (6). Al respecto, se ha reconocido la educación del paciente con DM como un componente esencial para fomentar el autocuidado, puesto que si conoce su patología y se encuentra informado se hace más fácil el tratamiento; en este sentido él mismo participará activamente en la prevención de las complicaciones por medio de sus cuidados (6).

De acuerdo con lo planteado anteriormente, la presente revisión se basa en la importancia del autocuidado y las intervenciones que lo fortalecen, ya que si se logra que los pacientes se informen y tomen las medidas pertinentes se evitará la aparición de complicaciones en su salud (7); por lo tanto se busca caracterizar la importancia de las intervenciones educativas para el autocuidado y prevención de pie diabético en personas con Diabetes Mellitus. Este concepto de autocuidado se fundamenta en la teoría de Dorothea E. Orem, quien lo considera como una función reguladora del hombre, la cual debe llevar a cabo de forma autónoma para conservar su salud y bienestar integral, con lo que se reafirma que es el objetivo de educación, para que las personas se hagan partícipes principales en su cuidado y mejoren su calidad de vida (8).

\section{Metodología}

La presente investigación es de tipo documental bajo el diseño de revisión narrativa de la literatura. Se elaboró en base a los componentes de la estrategia PICO, Población: Pacientes diagnosticados con DM; Intervención: intervenciones educativas frente a la prevención del pie diabético; Comparador: Atención habitual; Outcome/ desenlace: Nivel de autocuidado / prevalencia de pie diabético / prevalencia de amputaciones secundarias al pie diabético.

Se definieron como criterios de inclusión para los artículos los correspondientes a: estudios que evidencian las diferentes estrategias educativas de acuerdo a la estructura PICO planteada, todos los diseños metodológicos (cualitativos, cuantitativos y estudios secundarios) en idiomas español, inglés y en portugués, disponibles en los índices Ovid Nursing, LILACS, MEDLINE (PUBMED), Google Scholar y COCHRANE LIBRARY; además de los estudios encontrados en la literatura gris. Se excluyen los artículos publicados antes del año 2008.

Se realizó la búsqueda de los estudios por medio de las palabras clave "Diabetes Mellitus and Educative intervention and Diabetic foot and Self-care" en español, inglés y portugués. A continuación se procedió a la selección de los estudios, haciendo cribado y usando los criterios de inclusión y exclusión inicialmente en títulos y resúmenes; a continuación se eliminaron los duplicados y después se accedió al texto completo de los artículos pre - seleccionados. En la revisión de los textos completos se seleccionan 56 artículos a ser analizados (Ver figura 1 y Tabla 1).

\section{Análisis}

El análisis de los resultados se realizó por medio de la revisión crítica y síntesis de la literatura, identificando cuatro categorías emergentes que son relevantes para incentivar el autocuidado en la prevención del PD (Ver Figura 2, resumen y categorías de análisis), dichas categorías se mencionan a continuación. 


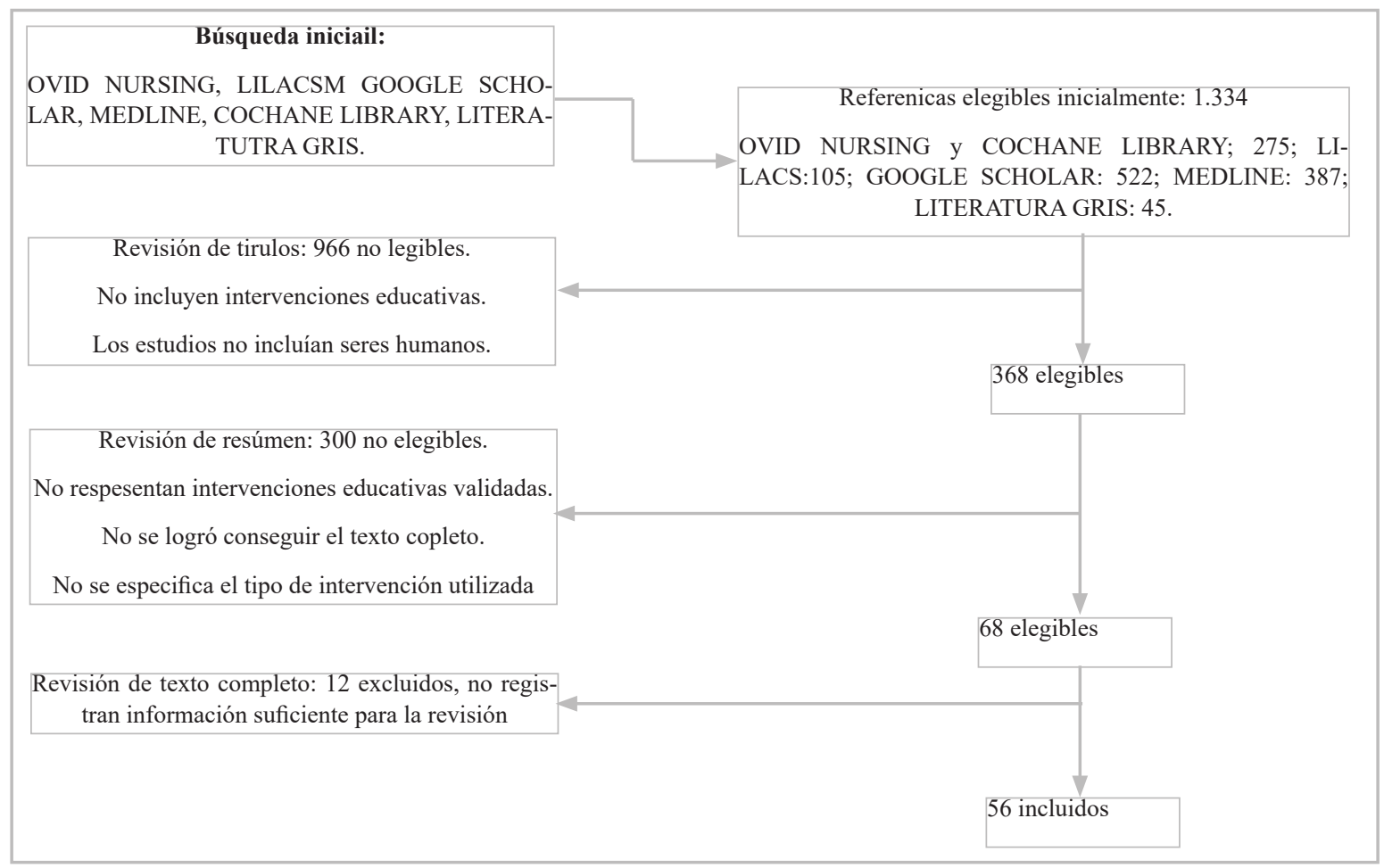

Figura 1. Flujograma selección de estudios

Fuente: Autoras

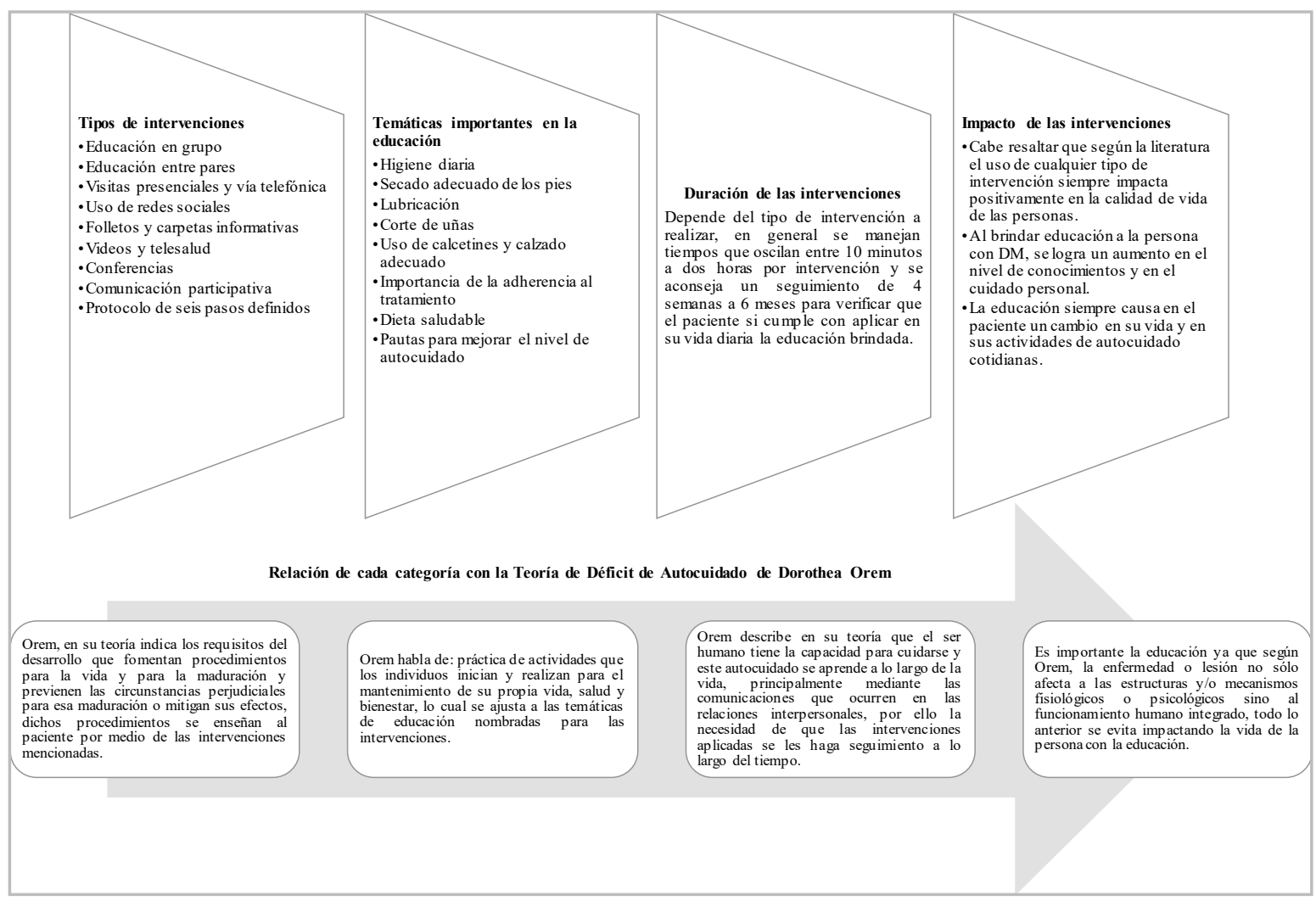

Figura 2: Categorías de análisis.

Fuente: Autoras 
Tabla 1: Estudios seleccionados

\begin{tabular}{cccc}
\hline Titulo & Diseño & Autores/Año & $\begin{array}{c}\text { Categoría a la que } \\
\text { aporta }\end{array}$ \\
\hline
\end{tabular}

Role of structured individual patient education in the prevention of vascular complications in newly diagnosed type 2 diabetes: The Individual Therapeutic Education in Newly Diagnosed type

2 diabetes (INTEND) randomized controlled.

Social media as a platform for information about diabetes foot care: a study of Facebook groups.

Acciones educativas de prevención del pie diabético.

Peer-to-peer education the pep talk: diabetes, healthy feet and you program in Canada.

Medium-term effectiveness of a group educational intervention on foot care in patients with type 2 diabetes.

"The group facilitates everything": meanings patients with type 2 diabetes mellitus assigned to health education groups.

Educational techniques in diabetes treatment

Grupo educação-apoio: visualizando o autocuidado com os pés de pessoas com diabetes mellitus.

Nurses' knowledge on diabetic foot ulcer disease and their attitudes towards patients affected: A cross-sectional institution-based study.

Atención de Enfermería en el paciente con pie diabético desde una perspectiva de autocuidado.

Mobile phone text messaging to improve knowledge and practice of diabetic foot care in a developing country: Feasibility and outcomes.

Improving rural and remote practitioners' knowledge of the diabetic foot: findings from an educational intervention.

Intervención educativa para mejorar la prevención del pie diabético en la atención primaria de salud.

Ensayo

controlado

aleatorio

Coppola A, Luzi L, Montalcini T, Giustina A, Gazzaruso C., 2018 (3)

2. Ítems a tratar en cada intervención

Revisión

Sistemática.

Estudio descriptivo transversal.

Estudio de caso.

Estudio descriptivo transversal.

Estudio descriptivo transversal.

\section{Investiga-}

ción cualitativa.

Estudio

narrativo.

Estudio descriptivo Transversal

Revisión de la literatura

Estudio correlacional

Diseño de estudio cuasi-experimental

Estudio descriptivo.
Hassan ZM., 2017 (17)

Abedin TA, Mamun M, Lasker M, Ahmed SW, Shommu N, Rumana N, Turin T., 2017 (8)

Vejerano-García P, Vejarano-García B, Duany-Amaro A, Vejerano-Duany A., 2009 (9)

Kuhnke JL, Rosenthal S, 2015 (10)

Ramón-Cabota J, Fernández-Trujillo M, Forcada-Vegab C, Guillem-Pera B., 2008 (11)

Pereira L, Antunes E., 2014 (12)

Selli L, Papaléo LK, Meneghel SN, Torneros JZ., 2005 (13)

Coelho MS, Silva DM, Guerreiro V., 2006. (14)

Kumarasinghe SA, Hettiarachchi P, Wasalathanthri S., 2018 (15).

González Salcedo P., 2008 (16)

Schoen D, Gausia K, Glance D, Thompson S., 2016 (18).

Barceló A, Robles S, White F, Jadue L, Vega J., 2001 (19)
4. Impacto de las intervenciones

1. Tipos de intervenciones

1. Tipos de intervenciones

1. Tipos de intervenciones

1. Tipos de intervenciones

4. Impacto de las intervenciones

1. Tipos de intervenciones

1. Tipos de intervenciones

1. Tipos de intervenciones

1. Tipos de intervenciones

3. Duración de las Intervenciones

4. Impacto de las intervenciones

1. Tipos de intervenciones

1. Tipos de intervenciones

1. Tipos de intervenciones

4. Impacto de las intervenciones 
Efectividad de un programa educativo sobre autocuidado en la prevención de complicaciones de miembros inferiores en pacientes con diabetes mellitus hospital Santa María del Socorro de ICA 2014-2015.

Are we telling the diabetic patients adequately about foot care?

Impacto de un programa institucional educativo en el control del paciente diabético.

Mejora en el control de los diabéticos tipo 2 tras una intervención conjunta: educación diabetológica y ejercicio físico.

Eficacia de un programa de educación sanitaria en la prevención y tratamiento de pacientes diagnosticados de Diabetes Mellitus que padecen Pie Diabético.

Autocuidado das pessoas com diabetes mellitus que possuem complicações em membros inferiores.

Efectividad de un programa educativo en pacientes con pie diabético de riesgo.

Cuidado com os pés diabéticos antes e após intervenção educativa.

Diabetes foot care education movies for Aboriginal people: Bran nue leg.

Comparison of three types of diabetic foot ulcer education plans to determine patient recall of education.

Role of telehealth in diabetic foot ulcer management.

Efectividad de intervención educativa en el conocimiento del paciente diabético sobre autocuidados.

Intervention mapping protocol for developing a theory-based diabetes self-management education program,
Revisión sistemática

Estudio

Huisacayna Díaz FM, Aguilar

Estudio de

Semino de Flores Y, Flores García caso.

AC, Ninahuaman Huisacayna LK, Yupanqui R., 2016 (20)

Estudio Ali R, Farooq U, Jalal-ud-din M

Transversal

Cuasi experimento

Ensayo

clínico

aleatorizado

controlado

Ensayo clínico aleatorizado.

Paniagua de Castro AV., 2017 (24)

Estudio

transversal

cuantitati-

vo.

Estudio

descriptivo.

Estudio casi

experimen-

tal.

Estudio de caso

Revisión

sistemática

de la litera-

tura.

Gomides D, Villas-Boas LC, Coelho AC, Pace AE., 2013 (25).

Pereira-Despaigne OL, Palay-Despaigne MS, Frómeta-Ríos V,

Neyra-Barrios RM., 2015 (26)

Pérez-Rodríguez MC, Godoy S, Mazzo A, Nogueira PC, Trevizan, MA, Mendes IAC., 2013 (27).

Australian Association of Health Promotion Professionals. 2016

(28).

Gravely SS, Hensley BK, Hagood-Thompson C, 2011 (29)

Singh TP, Vangaveti VN, Kennedy RL, Malabu UH., 2016 (30).

Chapman Sánchez M, García Almaguer R, Caballero González G, cuasi-experimental

Revisión de la literatura

Song M, Choi S, Kim SA, Seo K, Lee SJ, 2015 (32)
1. Tipos de intervenciones

1. Tipos de intervenciones

1. Tipos de intervenciones

1. Tipos de intervenciones

4. Impacto de las intervenciones

1. Tipos de intervenciones

1. Tipos de intervenciones

1. Tipos de intervenciones

4. Impacto de las intervenciones

1. Tipos de intervenciones

1. Tipos de intervenciones

4. Impacto de las intervenciones

1. Tipos de intervenciones

1. Tipos de intervenciones

4. Impacto de las intervenciones

1. Tipos de intervenciones

3. Duración de las

Intervenciones
2. Ítems a tratar en cada intervención 
Lower extremity ulcer management: best practice algorithm.

The importance of diabetes foot care education in a primary care setting.

A Health Education Pilot for Latina Women with Diabetes,

Compartilhando saberes e práticas de clientes com diabetes acerca dos cuidados com os pés para a prevenção de lesões: cuidado educativo de enfermagem.

Effects of Buerger Exercise Combined HealthPromoting Program on Peripheral Neurovasculopathy Among Community Residents at High Risk for Diabetic Foot Ulceration

Model of care for the diabetic foot.

Avaliação de um programa para prevenção de úlceras neuropáticas em portadores de diabetes.

Health education programmes to improve foot self-care practices and foot problems among older people with diabetes: a systematic review.

Culturally appropriate health education for type 2 diabetes mellitus in ethnic minority groups.

Patient education for preventing diabetic foot ulceration.

Promoção da saúde de pessoas com diabetes mellitus no cuidado educativo preventivo do pé-diabético

Prevention of foot ulcers in the at-risk patient with diabetes: a systematic review

Acurácia das intervenções de enfermagem para pacientes com diabetes mellitus tipo 2 em consulta ambulatoriala.

Prevention and management of diabetic foot ulcers

The efficacy of diabetic foot care education.

Prevention and management of neuropathic diabetic foot ulcers.
Revisión

sistemática

de la litera-

tura.

Estudio correlacional

Estudio

descriptivo

de corte

transversal

Estudio

cualitativo

Rodrigues RC., 2016 (36).

Coffman MJ, Ferguson BL, Steinman L, Talbot LA, Dunbar-Jacob J., 2012 (35)

Estudio cuasi-experimental

Chang CF, Chang CC, Hwang SL, Chen MY, 2015 (37)

2. Ítems a tratar en cada intervención

Reporte de
estudio

National Diabetes Programme

Ensayo clínico aleatorizado.

Revisión

sistemática

Estudio

de casos y

controles.

Revisión

sistemática

de la litera-

tura.

Estudio de métodos

mixtos eva-

luativo-in-

terpretativo.

Revisión

Sistemática

Estudio

prospecti-

vo, de corte

transversal

Revisión de la literatura

Estudio

Cualitativo

Estudio de caso.
Clinical Strategy and Programmes Directorate, 2011 (38)

Cisneros LL., 2010 (39)

Sharoni A, et al, 2016 (40).

Hawthorne K, Robles Y, Cannings-John R, Edwards AGK., 2008 (41).

Dorresteijn JAN, Kriegsman DMW, Assendelft WJJ, Valk GD., 2014 (42).

3. Duración de las Intervenciones

3. Duración de las Intervenciones

3. Duración de las Intervenciones

3. Duración de las Intervenciones

3. Duración de las Intervenciones

Santana da Silva LW, Souza Silva

J, Rossi Squarcini CF, Galvão

Souza F, Santos Ribeiro V, Ferreira Gonçalves D., 2016 (43)

Van Netten JJ, Precio PE, Lavery LA, Monteiro-Soares M, Rasmussen A, Jubiz Y, et al., 2016 (44)

3. Duración de las

3. Duración de las Intervenciones

Scain SF, Franzen E, Batista dos Santos L, Heldt E., 2013 (45)

Turns M., 2015. (46)

4. Impacto de las intervenciones

Nemcová J, Hlinková E., 2014 (47)

Jarrett L., 2013 (48) intervenciones Intervenciones
4. Impacto de las

4. Impacto de las intervenciones

4. Impacto de las intervenciones 
Atuação do enfermeiros da Estratégia Saúde da Família na prevenção do pé diabético Practice nurse family health strategy in the prevention of diabetic foot.

Development and validation of a diabetes foot self-care behavior scale.

Relationship between person's health beliefs and diabetes self-care management regimen.

Using a robot to personalise health education for children with diabetes type 1: A pilot study.

Modelos y teorías en enfermería: teoría del déficit de autocuidado.

Efectividad de una estrategia educativa sobre los parámetros bioquímicos y el nivel de conocimientos en pacientes diabéticos tipo 2 .

Importance of education in the prevention of diabetic foot syndrome.

La educación del paciente diabético con pie de riesgo.

Primary-care prevention activities in outpatients suffering from diabetic foot care.

Pacientes com diabetes mellitus: cuidados e prevenção do pé diabético em atenção primária à saúde

Effect of educational support on treatment adherence in patients with type 2 diabetes: An experimental study.

Factors related to self-care behaviours among patients with diabetic foot ulcers.

An educational intervention on foot self-care behaviour among diabetic retinopathy patients with visual disability and their primary caregivers

La educación del paciente diabético de debut para prevenir las úlceras del pie diabético
Estudio descriptivo, con abordaje cuantitativo.

Revisión de la literatura.

Estudio correlacional descriptivo.

Estudio analítico, exploratorio.

Revisión de la literatura

Cuasi-experimento

Artículo de revisión.

Estudio

prospectivo

cuasi-experimental

Estudio

descriptivo

de corte

transversal

Estudio

transversal cuantitativo

Estudio experimental

Estudio descriptivo de corte transversal

Estudio

cuasi-experimental

Estudio cuasi-experimental
Simplício de Oliveira P, Porto

Bezerra E, Lima de Andrade L,

Ferreira Gomes PL, Guimarães

Oliveira Soares MJ, Lopes Costa MM., 2016 (49)

Chin YF, Huang TT., 2013 (50)

4. Impacto de las intervenciones

Albargawi M, Snethen J, Al Gan4. Impacto de las nass A, Kelber S., 2017 (51) intervenciones

Blanson Henkemans OA, Bierman

BPB, Janssen J, Neerincx MA,

Looije R, Bosch HVD, et al., 2013 (52).

Berbiglia V, Banfield B., 2003 (53)

Pedraza Áviles AG, Martínez Vázquez R., 2007 (54)

Pokorna J., 2017 (55)

García Rodríguez, G., Victores

Moya, J. A., Mirabal Rodríguez,

A., Fleites Fonticiella, L., García

Rodríguez, Y., \& García Velázquez, J. M., 2017 (56)

Pinilla AE, Sánchez AL, Mejía A, Barrera MP., 2011 (57)

4. Impacto de las intervenciones

Saloum de Andrade NH, Sasso-Mendes KD, Gimenes Faria HT, Martins TA, Santos MA, Souza Teixeira CR, et al, 2010 (58)

Castro-Cornejo MA, Rico-Herrera L, Padilla-Raygoza N., 2014 (59)

Eun Jo Kim RN, MSc Kuem-Sun Han, 2020 (60)

Jiayu Li Lipei Gu Yujie Guo, 2019 (61)

García Rodríguez, G., Victores Moya, J. A., Mirabal Rodríguez, A., Fleites Fonticiella, L., García Rodríguez, Y., \& García Velázquez, J. M., 2020 (62)
4. Impacto de las intervenciones

4. Impacto de las intervenciones

4. Impacto de las intervenciones

4. Impacto de las intervenciones

4. Impacto de las intervenciones

Fuente: Elaborado por las autoras 


\section{Categoría 1: Tipo de intervenciones educativas}

A lo largo de la revisión se observó que existen diferentes intervenciones usadas para brindar educación en pro del autocuidado; además, se observó que para determinar de forma adecuada cuál o cuáles de los diferentes métodos se van a utilizar, se debe tener en cuenta las características de la población a tratar, identificando de forma minuciosa las necesidades del paciente, su nivel educativo y en general su situación particular; esto en función de que no siempre se van a tener las mismas necesidades en pacientes diferentes, así como en su capacidad para adaptar la información a su cotidianidad (9).

A continuación se explican los diferentes tipos de intervenciones. La primera es la Educación en grupos, la cual se destaca porque el $70 \%$ de los artículos analizados mencionan a este tipo de intervención. Es por eso que se considera según la literatura, una de las más efectivas, debido a que se permite la libre expresión de los pacientes en cuanto a sus creencias, sus métodos de cuidado y la efectividad de los mismos, convirtiéndose en una conversación compartida, en donde cada uno se expresa de forma libre; igualmente, tienen en cuenta la información de sus compañeros que consideran más importante, aplicándola a sus actividades diarias (9-12).

La segunda intervención es la Educación entre pares, que es un tipo de intervención similar a la anterior. Se maneja entre grupos de pacientes, quienes lideran y moderan las sesiones permitiendo que las personas compartan sus experiencias y refuercen sus conocimientos y aptitudes (13-15).

La tercera intervención analizada corresponde a las Visitas presenciales y visitas telefónicas. Estos dos métodos para intervenir a los pacientes son importantes, puesto que los pacientes con DM requieren de un cuidado especial que se realiza generalmente de forma personal; sin embargo, en algunas ocasiones y por motivos ajenos a la voluntad de las personas, ya que es difícil acceder a ella, razón por cual las visitas se pueden realizar vía telefónica. Estas intervenciones consisten en una interacción continua entre los pacientes y los profesionales de la salud, que se realiza con el fin de obtener datos relevantes acerca de las actividades que lleva a cabo el paciente para el cuidado de su salud; en este sentido el personal que educa busca las debilidades o el déficit que presenta el paciente en su autocuidado para fortalecerlo y mejorar su estado de salud (16-21).

La cuarta intervención consiste en el uso de Redes Sociales como Facebook, Twitter, entre otras, para realizar la distribución de materiales educativos a los pacientes, reforzando el tratamiento en general y el cuidado de los pies. De igual forma se puede contemplar el uso de aplicaciones telefónicas como el uso de WhatsApp o de foros en la red, a fin de enviar mensajes y recordatorios con información clara y concisa para el bienestar de las personas $(7,22-26)$. Dentro de esta modalidad de intervención también está el Uso de Videos, que se utilizan especialmente en población con bajo nivel educativo, ya que son muy ilustrativos y fáciles de comprender; también se los puede emplear en casos de pacientes escolares, ya que les facilita comprender la información para que la adopten en su vida (27-28).

La quinta intervención es la Comunicación Participativa, la cual favorece el aprendizaje y las conductas de autocuidado pedio en las personas con diabetes. El modelo de comunicación participativa abrió nuevos caminos de expresión e interacción, facilitando el intercambio y la creación de mensajes de acuerdo con las necesidades de estas personas (26). En este sentido los sistemas de telesalud y las conferencias son otras estrategias usadas que usan de la comunicación participativa permitiendo un empoderamiento por parte de los pacientes en función de un alto nivel de autocuidado $(16,29-30)$.

Finalmente, se encontró un Protocolo que consta de seis pasos, el mismo se recomienda en cinco estudios por ser ordenado y de fácil comprensión, debido al orden lógico y completo que conlleva. Los pasos a seguir son los siguientes: evaluar necesidades, definir los objetivos de rendimiento, identificar la teoría y los determinantes para el cambio de comportamiento general, desarrollar la matriz de objetivos de cambio, selección de métodos y estrategias para los objetivos de cambio, y por último la elaboración de un programa para llevar a cabo la educación de acuerdo a los objetivos (31).

Así, se evidencia la importancia de contar con tipos de intervenciones educativas que estén integradas en grupos de soporte social entre pares, que consideren los aspectos pedagógicos, los recursos didácticos y las características socio - culturales que requieren los adultos y adultos mayores con DM y sus cuidadores informales.

\section{Categoría 2: Temáticas a tratar en las intervenciones}

Luego de realizar la lectura y análisis de la información recolectada, se encontraron como temas importantes e infaltables en las intervenciones los siguientes: la ins- 
pección diaria de los pies, la realización de ejercicios para los pies, actividades como inculcar no caminar descalzo, uso de zapatos adecuados que sean suaves y cómodos, verificación del espacio interior de los zapatos antes de usarlos, uso de medias de algodón sin fisuras, corte adecuado de las uñas, apropiada hidratación de los pies, no utilizar bolsa de agua caliente en los pies, secado de los espacios interdigitales e importancia de la adherencia al tratamiento; estos son los cuidados que permitirán que las personas adopten medidas claras y específicas para evitar las complicaciones de la diabetes mellitus y en este caso puntual la prevención del pie diabético (3, 32-37).

Se debe señalar por tanto que estas intervenciones educativas aborden lo temas necesarios para que las personas con DM cuenten con un adecuado manejo integral de su estado de salud para garantizar un estado de bienestar; de igual manera hay que priorizar todos los aspectos prácticos que deben ser incorporados como parte de sus rutinas diarias de autocuidado para prevenir el PD.

\section{Categoría 3: Duración de las intervenciones educa- tivas}

Con respecto a la duración de las intervenciones, según los estudios hay una clara evidencia que a mayor tiempo se obtienen mejores resultados en general. En este sentido, se manejaron intervenciones que oscilaban entre diez minutos a dos horas diarias, durante un promedio de dos meses $(16,31,38-44)$. Sin embargo, las intervenciones que tuvieron una duración de dos semanas, al inicio eran satisfactorias, pero con bajo impacto ya que los pacientes volvían a perder el interés en la práctica de las actividades propias de su cuidado $(16,31,38$ 44). Por lo tanto, es importante que las intervenciones educativas sean continuas y extenderse en el tiempo, garantizando el seguimiento y reforzando las temáticas en los pacientes, para que se dé un impacto positivo y duradero y así evitar que se presenten complicaciones, ya sea por falta de información o que se brinde de prisa sin que tenga efecto en el tiempo (16, 31, 38-44).

Conviene subrayar que el tiempo ideal de duración de estas intervenciones educativas es variable, pues depende más del tipo de intervención y de las características propias de la persona con DM, ya que cada sujeto tiene formas distintas de aprender. No obstante, es necesario recalcar que estas intervenciones deben tener continuidad y sistematicidad en el tiempo; además, estar orga- nizadas en función del aporte de un equipo interdisciplinario que contribuya a mejorar todas las necesidades en salud de estos pacientes.

\section{Categoría 4: Impacto de las intervenciones en el nivel de autocuidado}

En general, la literatura analizada coincide en confirmar que al realizar cualquier tipo de intervención educativa de las anteriormente explicadas se van a obtener resultados positivos, ya que en todos los casos las personas presentan un mejor nivel de autocuidado. Situación que se presenta en grados diferentes dependiendo de la asertividad al elegir la intervención y de la duración o continuidad de la misma; es decir, que en los casos donde se brinda información por un corto tiempo se obtienen cambios parciales, en cambio si se da información que se prolongue en el tiempo, permitirá que los pacientes tengan un nivel de autocuidado mayor. $(8,12,16,19$, 23)

Como se ha dicho, una persona informada presenta un mayor autocuidado y una mejor calidad de vida, comparándolo con una que no tenga acceso a información veraz respecto a la prevención de las complicaciones. Los resultados anteriores ratifican la importancia de brindar información desde los primeros niveles de atención en salud y por personal preparado que identifique a los pacientes con DM, así como la intervención que se necesita para mejorar este tipo de autocuidado de forma rutinaria $(26,28,30,45-59)$. En definitiva, se necesita un mayor desarrollo de programas en este sentido, que además involucren la familia (60), a fin de que estas intervenciones educativas fomenten comportamientos positivos y efectivos de autocuidado (61).

En definitiva, las opciones con que cuenta el personal sanitario en la prevención de la diabetes mellitus son básicamente la promoción, la prevención, la curación y la rehabilitación; aunque últimamente se ha procurado tratar las alteraciones de salud especialmente con la promoción y la prevención. En tal sentido, se debe disponer de la herramienta básica para el control de la DM desde el momento en que se la diagnostica. Es por eso que la educación para la salud deba ser un proceso que se dé durante toda la vida para enseñar al individuo a conocer mejor su cuerpo y la forma adecuada de mantenerlo (62).

Para concluir, se puede indicar que el impacto que este tipo de intervenciones educativas generan en las per- 
sonas con DM es positivo y varía de acuerdo a la continuidad, contenido y aspectos pedagógicos que se incluyan. De ahí que algunas intervenciones generen una reflexión temporal en la persona, frente a las que tengan mayor impacto facilitando la incorporación de hábitos de autocuidado en la prevención del PD.

\section{Conclusiones}

Se logró cumplir el objetivo de analizar la producción científica actual relacionada con el tema de estudio, con los aportes de 56 estudios respecto a las cuatro categorías de análisis, así:

- Tipos de intervenciones, el 37\% de los artículos

- Ítems a tratar en cada intervención, solamente el $10 \%$ de los artículos lo señalaron. Fue la categoría con menor número de artículos; sin embargo, la información obtenida fue suficiente para la descripción planteada.

- Duración de las Intervenciones, el 13\% de los artículos

- Impacto de las intervenciones, el $41 \%$ de los artículos

En la síntesis analítica realizada a través de la presente revisión se identificaron las características que fundamentan las intervenciones educativas para el autocuidado y prevención de pie diabético en personas con DM. Al respecto, se evidencian las siguientes estrategias metodológicas: la educación en grupo, la educación entre pares, videoconferencias y uso de redes sociales. Estas estrategias al usarse de forma continua mejoran el nivel de autocuidado de las personas con DM y su calidad de vida; además, fortalecen las habilidades de autocuidado de esta población y generan un nivel de concientización que permite su incorporación como parte de sus rutinas diarias.

Del mismo modo, en la literatura revisada se propone que las personas con DM adquieran para su vida diaria una serie de habilidades que fortalezcan el autocuidado y que les facilite un adecuado manejo de su condición de salud, para evitar posibles complicaciones. A este respecto, entre las principales actividades que debe realizar el paciente con esta patología está la de adoptar estilos de vida saludables dentro de los que se incluye: una alimentación saludable, actividad física frecuente, consumo adecuado de medicamentos hipoglucemiantes orales o insulina y la adopción de cuidados rutinarios de los pies (auto monitoreo, técnica de limpieza adecuada, uso de calzado con medidas especiales, corte de uñas, etc.).

Teniendo en cuenta toda la información descrita se recomienda que los profesionales de la salud, especialmente los de enfermería, elaboren planes de cuidado que incluyan actividades de promoción y prevención por medio de intervenciones educativas como parte de la atención sanitaria habitual. Acciones que se deben llevar a cabo con el fin de brindar un mejor servicio a los pacientes, promover su autocuidado y evitar que presenten complicaciones en su salud. Con estas medidas se espera que como cuidadores de la salud, los enfermero/as se enfoquen en enseñar al paciente su rutina de cuidado diario a fin de evitar y/o retardar la aparición de complicaciones de la DM, con especial énfasis en el PD.

\section{Agradecimientos}

A la Universidad de Boyacá por la disposición para facilitar el desarrollo del proyecto.

\section{Conflicto de intereses}

Se declara que no existe ningún conflicto de interés por parte de las autoras.

\section{Financiación}

Se declara que la financiación es de la Universidad de Boyacá en cuanto a los recursos para el desarrollo de este estudio.

\section{Referencias Bibliográficas}

1. Ramírez-Roca LA, Palencia-Prado J, Castro-Martínez MG. Revisión de las guías de tratamiento farmacológico de diabetes mellitus tipo 2 y opinión en Centroamérica. Med Int Méx. 2015;31(6):733-748.

2. Marinel R, Blanes M, Escudero R, Ibáñez E, Rodríguez O. Tratado del pie diabético. Madrid: Jarpyo; 2002.

3. Coppola A, Luzi L, Montalcini T, Giustina A, Gazzaruso C. Role of structured individual patient education 
in the prevention of vascular complications in newly diagnosed type 2 diabetes: the Individual Therapeutic Education in Newly Diagnosed type 2 diabetes (INTEND) randomized controlled trial. Endocrine. 2018; 60(1);46-49. https://doi.org/10.1007/s12020-017-1427-x

4. Menezes L, Guedes MV, Moura N, Oliveira R, Vieira L, Barros A. Estratégias educativas para pessoas diabéticas com pé em risco neuropático: síntese de boas evidências. REE -Bra-. 2016:81-16. https://doi. org/10.5216/ree.v18.40281

5. Carrillo-Esper R, Muciño-Bermejo MJ. Tratamiento quirúrgico de la diabetes mellitus tipo 2. Med Int Méx. 2012;28(1):219-230.

6. Acosta S. et al. Programa educativo para la prevención y atención de la diabetes mellitus en estudiantes y trabajadores de la Universidad de Cienfuegos. Conrado, 2019, 15 (69): 19-25.

7. Aalaa M, Sanjari M, Shahbazi S, Shayeganmehr Z, Abooeirad M, Amini MR, et al. Diabetic foot workshop: Improving technical and educational skills for nurses. Med J Islam Repub Iran. 2017;31(1):40-42. https://doi.org/10.18869/mjiri.31.8

8. Abedin TA, Mamun M, Lasker M, Ahmed SW, Shommu N, Rumana N, Turin T. Social Media as a Platform for Information About Diabetes Foot Care: A Study of Facebook Groups. Can J Diabetes. 2017;41(1):97101. https://doi.org/10.1016/j.jcjd.2016.08.217.

9. Vejerano-García P, Vejarano-García B, Duany-Amaro A, Vejerano-Duany A. Acciones educativas de prevención del pie diabético. Red Hum Méd. 2009; 9(2):1-20.

10. Kuhnke JL, Rosenthal S. Peer-to-Peer Education: The PEP Talk: Diabetes, Healthy Feet and You Program in Canada. J Wound Ostomy Continence Nurs. 2015;42(4):321-323. https://doi.org/10.1097/ WON.0000000000000155.

11. Ramón-Cabota J, Fernández-Trujillo M, Forcada-Vegab C, Guillem-Pera B. Medium-term effectiveness of a group educational intervention on foot care in patients with type 2 diabetes. Enferm Clínica. 2008;18(6):302-308. https://doi.org/10.1016/S1130-8621(08)75852-2

12. Pereira L, Antunes E. "The group facilitates everything": meanings patients with type 2 diabetes mellitus assigned to health education groups. Rev Latino-Am Enfermagem. 2014;22(6):980-987. https://doi. org/10.1590/0104-1169.0056.2506

13. Selli L, Papaléo LK, Meneghel SN, Torneros JZ. Técnicas educacionales en el tratamiento de la diabetes. Cad Saúde Pública. 2005;21(5):1366-1372. https://doi.org/10.1590/S0102-311X2005000500008

14. Coelho MS, Silva DM, Guerreiro V. Grupo educação-apoio: visualizando o autocuidado com os pés de pessoas com diabetes mellitus. Ciên Cuid Saúde. 2006;5(1):11-15.

15. Kumarasinghe SA, Hettiarachchi P, Wasalathanthri S. Nurses' knowledge on diabetic foot ulcer disease and their attitudes towards patients affected: A cross-sectional institution-based study. J Clin Nurs. 2018; 27:203-212. https://doi.org/10.1111/jocn.13917

16. González Salcedo P. Atención de Enfermería en el paciente con pie diabético desde una perspectiva de autocuidado. Investig Enferm Imagen Desarro. 2008;10(2):63-95.

17. Hassan ZM. Mobile phone text messaging to improve knowledge and practice of diabetic foot care in a developing country: Feasibility and outcomes. Int J Nurs Pract. 2017;23(S1):1-6. https://doi.org/10.1111/ ijn.12546

18. Schoen D, Gausia K, Glance D, Thompson S. Improving rural and remote practitioners' knowledge of the diabetic foot: findings from an educational intervention. J Foot Ankle Res. 2016;9(26):1-11. https://doi. org/10.1186/s13047-016-0157-2

19. Barceló A, Robles S, White F, Jadue L, Vega J. Una intervención para mejorar el control de la diabetes en 
Chile. Rev Panam Salud Pública. 2001;10(5):328-333.

20. Huisacayna-Díaz FM, Aguilar-Semino de Flores Y, Flores-García AC, Ninahuaman-Huisacayna LK, Yupanqui R. Efectividad de un programa educativo sobre autocuidado en la prevención de complicaciones de miembros inferiores en pacientes con diabetes mellitus Hospital Santa María del Socorro de ICA 20142015. Rev Enferm Vanguard. 2016;4(2):41-47. https://doi.org/10.35563/revan.v4i2.228

21. Ali R, Farooq U, Jalal-ud-din M, Jadoon RJ, Alam MA, Qureshi A, et al. Are we telling the diabetic patients adequately about foot care? J Ayub Med Coll Abbottabad. 2016;28(1):161-163.

22. Mendoza-Romo MA, Velasco-Chávez JF, Nieva de Jesús RN, Andrade-Rodríguez HJ, Rodríguez-Pérez $\mathrm{CV}$, Palou-Fraga E. Impacto de un programa institucional educativo en el control del paciente diabético. Rev Med Inst Mex Seguro Soc. 2013;51(3):254-259.

23. Ariza-Copado C, Gavara-Palomara V, Muñoz-Ureña A, Aguera Menguala F, Soto-Martínez M, Lorca Serralta JR. Mejora en el control de los diabéticos tipo 2 tras una intervención conjunta: educación diabetológica y ejercicio físico. Aten Primaria. 2011;43(8):398-406. https://doi.org/10.1016/j.aprim.2010.07.006

24. Paniagua-de-Castro AV. Eficacia de un programa de educación sanitaria en la prevención y tratamiento de pacientes diagnosticados de Diabetes Mellitus que padecen Pie Diabético. [Trabajo fin de Máster]. [Internet]. Cantabria, España: Universidad de Cantabria; 2017 [citado 19 Mar 2018]. Disponible en: https:// repositorio.unican.es/xmlui/bitstream/handle/10902/12406/PaniaguadeCastroA.pdf?sequence $=1$

25. Gomides D, Villas-Boas LC, Coelho AC, Pace AE. Autocuidado das pessoas com diabetes mellitus que possuem complicações em membros inferiores. Acta Paul Enferm. 2013;26(3):289-293. https://doi. org/10.1590/S0103-21002013000300014

26. Pereira-Despaigne OL, Palay-Despaigne MS, Frómeta-Ríos V, Neyra-Barrios RM. Efectividad de un programa educativo en pacientes con pie diabético de riesgo. Medisan. 2015; 19(1):69-77

27. Pérez-Rodríguez MC, Godoy S, Mazzo A, Nogueira PC, Trevizan, MA, Mendes IAC. Cuidado com os pés diabéticos antes e após intervenção educativa. Enferm Global. 2013;12(29):43-52.

28. Australian Association of Health Promotion Professionals. Diabetes foot care education movies for Aboriginal people: Bran nue leg. Promotor de Salud J Austr. 2016;27(1):88-89. https://doi.org/10.1071/ HE15040

29. Gravely SS, Hensley BK, Hagood-Thompson C. Comparison of three types of diabetic foot ulcer education plans to determine patient recall of education. J Vasc Nurs. 2011;29(3);113-9.https://doi.org/10.1016/j. jvn.2011.06.001

30. Singh TP, Vangaveti VN, Kennedy RL, Malabu UH. Role of telehealth in diabetic foot ulcer management-A systematic review. Aust J Rural Health. 2016;24(4):224-229. https://doi.org/10.1111/ajr.12284

31. Chapman-Sánchez M, García-Almaguer R, Caballero-González G, Paneque-Caballero Y, Sablón-Mariño A. Efectividad de intervención educativa en el conocimiento del paciente diabético sobre autocuidados. Revi Cuba Enferm. 2016;32(1):49-59.

32. Song M, Choi S, Kim SA, Seo K, Lee SJ. Intervention mapping protocol for developing a theory-based diabetes self-management education program. Res Theory Nurs Pract. 2015;29(2):94-112.

33. Simms KW, Ennen K. Lower extremity ulcer management: best practice algorithm. J Clin Nurs. 2011;20:8693. https://doi.org/10.1111/j.1365-2702.2010.03431.x

34. Formosa C, Gatt A, Chockalingam N. The importance of diabetes foot care education in a primary care setting. J Diabetes Nurs. 2012;16(10):410-414.

35. Coffman MJ, Ferguson BL, Steinman L, Talbot LA, Dunbar-Jacob J. A Health Education Pilot for Latina Women with Diabetes. Clin Nurs Res. 2012;22(1):70-81. https://doi.org/10.1177/1054773812451746 
36. Rodrigues RC. Compartilhando saberes e práticas de clientes com diabetes acerca dos cuidados com os pés para a prevenção de lesões: cuidado educativo de enfermagem [tesis]. Brasil: Universidade Federal do Rio de Janeiro; 2016.

37. Chang CF, Chang CC, Hwang SL, Chen MY. Effects of Buerger Exercise Combined Health-Promoting Program on Peripheral Neurovasculopathy Among Community Residents at High Risk for Diabetic Foot Ulceration. Worldviews on Evidence-Based Nursing. 2015;12(3): 145-153. http://doi.org/10.1111/ $\underline{\text { wvn. } 12091}$

38. National Diabetes Programme Clinical Strategy and Programmes Directorate. Model of care for the diabetic foot [Internet] 2011 [citado 2 Mar 2018] Disponible en: http://www.podiatryireland.com/attachments/9998eba0-e877-48a5-8cda-42db7bd7a8ec.PDF

39. Cisneros LL. Avaliação de um programa para prevenção de úlceras neuropáticas em portadores de diabetes. Braz J Phys Ther [Internet]. 2010 [citado 17 Mar 2018];14(1). Disponible en: http://pesquisa.bvsalud. org/portal/resource/es/lil-552823

40. Sharoni A, Sakdiah H, Mohd N, Baharm DA. Health education programmes to improve foot self-care practices and foot problems among older people with diabetes: a systematic review. Int J Older People Nurs [Internet]. 2016 [citado 17 Mar 2018];11(3):214-239. Disponible en: https:/onlinelibrary.wiley.com/ doi/full/10.1111/opn.12112

41. Hawthorne K, Robles Y, Cannings-John R, Edwards AGK. Culturally appropriate health education for type 2 diabetes mellitus in ethnic minority groups. Cochrane Database of Systematic Reviews. 2008;(3) CD006424. http://doi.org/10.1002/14651858.CD006424.pub2

42. Dorresteijn JAN, Kriegsman DMW, Assendelft WJJ, Valk GD. Patient education for preventing diabetic foot ulceration. Cochrane Database of Systematic Reviews. 2014; (12)CD001488. http://doi. org/10.1002/14651858.CD001488.pub5

43. Santana-da-Silva LW, Souza-Silva J, Rossi-Squarcini CF, Galvão-Souza F, Santos-Ribeiro V, Ferreira-Gonçalves D. Promoção da saúde de pessoas com diabetes mellitus no cuidado educativo preventivo do pé-diabético. Cienc Enferm [Internet]. 2016 [citado 17 Mar 2018]. Disponible en: https://scielo.conicyt.cl/ scielo.php?script $=$ sci arttext\&pid $=$ S0717-95532016000200008

44. Van-Netten JJ, Precio PE, Lavery LA, Monteiro-Soares M, Rasmussen A, Jubiz Y, et al. Prevention of foot ulcers in the at-risk patient with diabetes: a systematic review. Diabetes Metab Res Rev [Internet]. 2016 [citado 18- Mar 2018];32(S1):84-98. Disponible en: https://www.ncbi.nlm.nih.gov/pubmed/26340966

45. Scain SF, Franzen E, Batista dos Santos L, Heldt E. Acurácia das intervenções de enfermagem para pacientes com diabetes mellitus tipo 2 em consulta ambulatorial. Rev Gaúcha Enferm. 2013;34(2):14-20. http://dx.doi.org/10.1590/S1983-14472013000200002

46. Turns M. Prevention and management of diabetic foot ulcers. Br J Community Nurs [Internet]. 2015 [citado 17 Mar 2018];20(Sup3):S30-S37. Disponible en: https://www.magonlinelibrary.com/doi/abs/10.12968/ bjen.2015.20.Sup3.S30

47. Nemcová J, Hlinková E. The efficacy of diabetic foot care education. J Clin Nurs. 2014;23:877-882. http://doi.org/doi:10.1111/jocn.12290

48. Jarrett L. Prevention and management of neuropathic diabetic foot ulcers. Nurs Stand [Internet]. 2013 [citado 17 Mar 2018];28(7):55. Disponible en: https://journals.rcni.com/doi/abs/10.7748/ns2013.10.28.7.55. $\underline{\mathrm{e} 7346}$

49. Simplício-Oliveira P, Porto-Bezerra E, Lima-de-Andrade L, Ferreira-Gomes PL, Guimarães-Oliveira Soares MJ, Lopes-Costa MM. Atuação do enfermeiros da Estratégia Saúde da Família na prevenção do pé diabético Practice nurse family health strategy in the prevention of diabetic foot. J Res Fundam Care on- 
line [Internet]. 2016 Jul 15 [citado 17 Mar 2018];8(3):4841-4849. Disponible en: http://www.seer.unirio. br/index.php/cuidadofundamental/article/view/4398

50. Chin YF, Huang TT. Development and validation of a diabetes foot self-care behavior scale. J Nurs Res [Internet] 2013 [citado 17 Mar 2018];21(1):19-25. Disponible en: https://journals.lww.com/jnrtwna/Fulltext/2013/03000/Development_and_Validation_of_a_Diabetes_Foot.5.aspx

51. Albargawi M, Snethen J, Al Gannass A, Kelber S. Relationship between person's health beliefs and diabetes self-care management regimen. J Vasc Nurs. 2017;35(4):187-192. https://doi.org/10.1016/j. jvn.2017.07.002

52. Blanson-Henkemans OA, Bierman-BPB, Janssen J, Neerincx-MA, Looije R, Bosch HVD, et al. Using a robot to personalise health education for children with diabetes type 1: A pilot study. Patient Educ Couns. 2013;92(2):174-181. https://doi.org/10.1016/j.pec.2013.04.012

53. Berbiglia V, Banfield B. Modelos y teorías en enfermería: teoría del déficit de autocuidado. 7 ed. Barcelona: Elsevier Mosly; 2003.

54. Pedraza-Áviles AG, Martínez-Vázquez R. Efectividad de una estrategia educativa sobre los parámetros bioquímicos y el nivel de conocimientos en pacientes diabéticos tipo 2. Rev Endocrinol Nutr [Internet]. 2007 Jul - Sep [citado 18 Mar 2018];15(3):165-174. Disponible en: http://www.medigraphic.com/pdfs/ endoc/er-2007/er073g.pdf

55. Pokorna J. Importance of education in the prevention of diabetic foot syndrome. Neuro Endocrinol Lett [Internet]. 2017 [citado 18 Mar 2018]; 38(4):255-256. Disponible en: https://www.ncbi.nlm.nih.gov/pub$\underline{\operatorname{med} / 28871710}$

56. García-Velásquez JM, Brito-Blanco D, Santos-Rofes BI, Ricardo-Olivera D, García-Albelo E, Fleites-Fonticiella L. La educación del paciente diabético con pie de riesgo. Acta Médica del Centro [Internet]. 2017 [citado 19 Mar 2018];12(1):29-37. Disponible en: http://www.medigraphic.com/pdfs/medicadelcentro/ mec-2018/mec181d.pdf

57. Pinilla AE, Sánchez AL, Mejía A, Barrera MP. Primary-care prevention activities in outpatients suffering from diabetic foot care. Rev Salud Pública [Internet]. 2011 [citado 17 Mar 2018];13(2):262-273. Disponible en: http://pesquisa.bvsalud.org/portal/resource/es/lil-602873

58. Saloum de Andrade NH, Sasso-Mendes KD, Gimenes-Faria HT, Martins TA, Santos MA, Souza-Teixeira $\mathrm{CR}$, et al. Pacientes com diabetes mellitus: cuidados e prevenção do pé diabético em atenção primária à saúde. Rev Enferm UERJ Rio de Janeiro [Internet]. 2010 Out - Dez [citado 17 Mar 2018];18(4):616-621. Disponible en: http://saudepublica.bvs.br/pesquisa/resource/pt/bde-19986

59. Castro-Cornejo MA, Rico-Herrera L, Padilla-Raygoza N. Effect of educational support on treatment adherence in patients with type 2 diabetes: An experimental study. Enferm Clínica. 2014 May - Jun;24(3):162167. https://doi.org/10.1016/j.enfcli.2013.11.004

60. Eun Jo Kim RN, MSc Kuem-Sun Han. Factors related to self-care behaviours among patients with diabetic foot ulcers. Juurnal Of Clinica Nursing, 2020, 29 (9): 1712-1722. https://doi.org/10.1111/jocn.1521

61. Jiayu Li, Lipei Gu, Yujie Guo. An educational intervention on foot self-care behaviour among diabetic retinopathy patients with visual disability and their primary caregivers. 2019, 28(13):2506-2516. https:// doi.org/10.1111/jocn.14810

62. García-Rodríguez G, Victores-Moya JA, Mirabal-Rodríguez A, Fleites-Fonticiella L, García-Rodríguez Y, García Velázquez JM. La educación del paciente diabético de debut para prevenir las úlceras del pie diabético. Acta Médica del Centro, 2020, 14(1), 68-81. https://www.medigraphic.com/cgi-bin/new/resumen. cgi?IDARTICULO $=92223$ 